Agrimensura, Vol. 2, No. 6, p.20, 1996

\title{
Appropriate Cadastral Systems
}

Ian Williamson

Chairperson, Commission 7 (Cadastre and Land Management)

International Federation of Surveyors

Professor of Surveying and Land Information

Department of Geomatics

The University of Melbourne

Parkville, Melbourne

Australia 3052

Tel: $+61-3-93444431$

Fax: $+61-3-93474128$

Email: i.williamson@eng.unimelb.edu.au

Cadastral systems are not ends in themselves and support effective land markets, increased agricultural productivity, sustainable economic development, environmental management, political stability and social justice, although it is absolutely essential that each cadastral system is designed appropriately to serve the needs of the individual country.

Cadastral reform must focus on the key processes which are associated with adjudicating, transferring and sub-dividing land rights, not just the concept of a cadastre or the individual activities of title registration or cadastral surveying.

There is a vast array of legal, technical, administrative and institutional options available in designing and establishing an appropriate cadastral system, providing a continuum of forms of cadastre ranging from the very simple to the very sophisticated. Such flexibility allows cadastres to record a continuum of land tenure arrangements from private and individual land rights through to communal land rights, as well as having the ability to accommodate traditional or customary land rights.

The success of a cadastral system is not dependent on its legal or technical sophistication, but whether it protects land rights adequately and permits those rights to be traded (where appropriate) efficiently, simply, quickly, securely and at low cost. However if the resources are not available to keep the cadastral system up-to-date then there is little justification for its establishment.

Some of the important lessons that have been learnt from establishing cadastral systems include:

- Efficiency ultimately requires formal recognition of individual land rights and the establishment of cadastral systems, where population densities cause land to be scarce, as farming becomes more commercialised, as farming technologies improve and as land markets emerge.

- The design of cadastral systems must be systematic, sustainable and sensitive to the culture, needs, resources and level of development of individual countries. Cadastres should be designed for the needs of land holders, not central government. They should be decentralised to the local or village level.

- The importance of an appropriate base map for the cadastral system which can also be used for many other purposes. However the cadastral system must be designed with the land registers having equal importance to the cadastral map.

- The appropriate use of modern technologies which are used to support the establishment and maintenance of cadastral systems, noting that while the use of computers, for example, may speed 
up some activities, such as the creation and maintenance of cadastral indexes, it may also slow down others, such as the creation of a base map. It must be remembered that effective and efficient land markets based on modern cadastral systems do not require computerised cadastral maps.

- cadastral systems should be designed with a national focus, but should be sufficiently flexible to accommodate both urban and rural requirements.

Cadastral reform should focus on what is the most appropriate cadastral system to serve the needs of an individual country and must recognise that no two cadastres will be the same. Unless cadastral systems are appropriate to the circumstances and requirements of the country for which they are designed, they can do more harm than good!

Ian Williamson

\section{Sistemas de Catastro Apropiados}

Ian Williamson

Chairperson, Commission 7 (Cadastre and Land Management)

International Federation of Surveyors

Professor of Surveying and Land Information

Department of Geomatics

The University of Melbourne

Parkville, Melbourne

Australia 3052

Tel: $+61-3-93444431$

Fax: $+61-3-93474128$

Email: i.williamson@eng.unimelb.edu.au

Sistemas de catastro no sòlo estàn limitados a desarrollar tareas de catastro, sino que cumplen una funciòn importante en administraciòn de tierras en el mercado actual, por ejemplo, aumentar producciòn agrìcola, sustentar desarrollo econòmico, mejor administraciòn ambiental, estabilidad polìtica y justicia social, sin embargo es absolutamente esencial que cada sistema de cadastro sea diseñado apropiadamente para cumplir con las necesidades de cada paìs.

Reformas en catastro tienen que ser enfocadas en procesos claves asociados con la adjudicaciòn, transferencia y subdivisiòn de derechos de tierras, no solamente se toma en cuenta el concepto de catastro en sì, sino que incluye otras ramas aparte de registro de tìtulos y agrimensura.

En el diseño de un sistema de catastro se tiene que tomar en cuenta una gran cantidad de asuntos legales, tècnicos, administrativos e institucionales, para proveer en forma continua servicios de catastro, desde lo mas simple a lo mas sofisticado. Esta flexibilidad permite una relaciòn contìnua en la clasificacion de bienes raices y sus propietarios, desde derechos privados e individuales hasta derechos comunitarios, $\mathrm{y}$ debe tambièn contar con la habilidad para acomodar derechos y costumbres tradicionales.

El èxito de un sistema de catastro no depende de su sofisticaciòn, de sus legalidades o tecnicalidades, sino que de su capasidad de protejer derechos de tierras adecuadamente y permitir que estos derechos puedan ser comercializados en forma simple, eficiente, ràpida, segura y a bajo costo. Sin embargo, si los recursos no estan al alcance para mantener el sistema de catastro al dia, no se justifica la formaciòn de èste.

Algunas de las lecciones mas importantes que hemos aprendido formando o diseñando sistemas de catastro son: 
- Eficiencia requiere un reconocimiento formal de derechos de tierras individuales y el establecimiento de sistemas de catastro en lugares donde la densidad de poblaciòn es tal que la tierra se hace escasa, a medida que la agricultura se comercializa, a medida que nuevas tecnologìas agrarias son implementadas y nuevos mercados de bienes raices surgen.

- El diseño de un sistema de catastro tiene que ser sistemàtico, sustentable, sensible a nivel cultural, nececidades, recursos y nivel de desarrollo de cada paìs. Sistemas de catastro deberian ser diseñados para satisfacer las necesidades de propietarios y no de un gobierno central. Tiene que ser un sistema decentralisado a nivel local.

- La importancia de contar con un mapa base o matrìs apropiado para catastro y otros propòsitos. Sin embargo el sistema de catastro tiene que ser diseñado tomando en cuenta el sistema de registro de bienes raises y darle la misma importancia que al mapa de catastro.

- El uso apropiado de tecnologia moderna para el diseño, uso y mantenciòn de sistemas de catastro, por ejemplo, tomando en cuenta que el uso de computadoras podrìa acelerar algunas actividades, como la creaciòn y mantenciòn de ìndices de clasificaciòn, pero puede retardar la creaciòn de mapas bases o matrices. Tenemos que recordar que un mercado de bienes raices efectivo y eficiente basado en sistema de catastro modernos no necesitan mapas de catastro computarisados.

- Sistemas de catastro deberian ser diseñados con un foco nacional, pero suficientemente flexibles para proveer soluciones a nivel urbano y rural.

Cualquier reforma deberìa ser dedicada al desarrollo de el sistema de catastro mas apropiado para satisfacer las necesidades individuales de cada paìs y se tiene que reconocer que no existen dos sistemas de catastro iguales, es decir, cada sistema es ùnico. A no ser que los sistemas de catastro sean apropiados para ciertas circunstancias y requerimientos de cada paìs, estos pueden producir mas problemas que soluciones.

Ian Williamson 


\section{University Library}

\section{- M M N E R VA A gateway to Melbourne's research publications}

Minerva Access is the Institutional Repository of The University of Melbourne

Author/s:

WILLIAMSON, IAN

Title:

Appropriate cadastral systems

Date:

1996

Citation:

Williamson, I. (1996). Appropriate cadastral systems. Agrimensura, 2(6).

Publication Status:

Published

Persistent Link:

http://hdl.handle.net/11343/34006 